\title{
Characterization of bacteriophages used in the Salmonella enterica serovar Enteritidis phage- typing scheme
}

Correspondence

N. De Lappe

nialldinsrl@netscape.net

Received 2 January 2008

Accepted 15 September 2008

\author{
N. De Lappe, ${ }^{1,2}$ G. Doran, ${ }^{2}$ J. O'Connor, ${ }^{2}$ C. O'Hare ${ }^{2}$ and M. Cormican ${ }^{1,2}$ \\ ${ }^{1}$ National University of Ireland Galway, Galway, Republic of Ireland \\ ${ }^{2}$ National Salmonella Reference Laboratory, Galway, Republic of Ireland
}

The 16 Salmonella enterica serovar Enteritidis (S. Enteritidis) typing phages (SETPs) used in the Laboratory of Enteric Pathogens (Health Protection Agency, London, UK) phage-typing scheme have not previously been characterized in detail. We have examined the adsorption properties of the phages with respect to a number of $S$. enterica serovars and defined phage morphology with electron microscopy. PFGE was used to estimate overall genome size and banding patterns generated by electrophoresis following restriction endonuclease digestion of the genome with Hindlll were compared. PCR amplification and sequencing of selected genes was performed. The 16 phages comprise three morphotypes, Podoviridae (SETP1, 8, 10, 14, 15 and 16),

Siphoviridae (SETP3, 5, 7, 11, 12 and 13) and Myoviridae (SETP2, 4, 6 and 9). All Podoviridae and Siphoviridae, but not Myoviridae, adsorbed to the $\mathrm{O} 12$ lipopolysaccharide antigen of Salmonella serogroups $B(4,12)$ and $D_{1}(9,12)$. The genome sizes for the Podoviridae and Siphoviridae (PFGE-A) were approximately $42 \mathrm{~kb}$. The genome size for Myoviridae SETP2, 4 and 9 was $36.5 \mathrm{~kb}$, and for myovirus SETP6 was $27 \mathrm{~kb}$. Hindlll digestion of phage DNA produced 9 distinct patterns of 8 to 11 bands. Relationships between phages based on digest patterns were consistent with those defined by morphology. The Podoviridae had homologues of several P22 genes while the Siphoviridae had homologues of several genes present in the sequenced siphovirus SETP3 (EF177456). This study represents an initial step in characterizing the molecular basis that underlies the widely used $S$. Enteritidis typing scheme.

\section{INTRODUCTION}

Salmonella enterica serovar Enteritidis ( $S$. Enteritidis) is the most common Salmonella serotype infecting humans worldwide. This serotype is endemic in poultry and can contaminate eggs from hens that are healthy (Porwollik et al., 2005). In Europe phage typing using the scheme of the Laboratory of Enteric Pathogens (LEP) of the Health Protection Agency (London, UK) is widely used for subtyping S. Enteritidis (Ward, 2006). This scheme subdivides S. Enteritidis into 74 phage types (PTs) based on reactions with 16 phages (originally 10 phages with 6 subsequent additions).

The history of the initial $10 \mathrm{~S}$. Enteritidis typing phages (SETPs) has been published (Ward et al., 1987). Most typing phages originate from lysogenic Salmonella isolates

Abbreviations: LEP, Laboratory of Enteric Pathogens; PT, phage type; SETP, Salmonella enterica serovar Enteritidis typing phage.

The GenBank/EMBL/DDBJ accession numbers for the gene sequences of the Salmonella phages are EF151167-EF151189 and EF212157-EF21277.

Tables of primers, phage typing and induction data, and sequence data are available as supplementary material with the online version of this paper. or from sewage. Some of the typing phages were developed from the original isolates by propagation in different hosts to alter their plating properties, a process known as adaptation. The history of the additional six phages has not been formally published.

There is extensive experience in the application of phage typing to epidemiological studies. Phage-typing is discriminatory, and a high degree of inter-laboratory agreement can be achieved with standardization of methods and reagents (Raes et al., 2000). However, the molecular basis of the phage-host interaction that determines PT is not well understood.

Most research on specific bacterial resistance mechanisms to phages has been performed by microbiologists in the dairy industry. The factors identified in this research include receptors for phages on the bacterial cell wall, various bacterial restriction-modification systems and abortive infection mechanisms (Fortier et al., 2005). The presence of viable or defective prophages encoding immunity regions, superinfection exclusion systems and antigen-altering genes may also inhibit attack by other phages (Vander Byl \& Kropinski, 2000). 
A range of factors have been suspected or confirmed as impacting on phage reaction patterns in $S$. Enteritidis. The loss of lipopolysaccharide from PT1, PT4 and PT6 isolates resulted in their conversion to PT7 (Baggesen et al., 1997), and differences in core lipopolysaccharide structure have been postulated to account for much of the variations in reactions with the phage typing panel (Guard-Petter, 1999). Lysogenization with typing phages (Rankin \& Platt, 1995) and acquisition of plasmids (Frost et al., 1989; Ridley et al., 1996) resulted in loss of sensitivity to certain phages and change of PT.

Several S. enterica serovar Typhimurium (S. Typhimurium) phages, including ST64T (Mmolawa et al., 2003) and ST104 (Tanaka et al., 2004), share widespread sequence similarity with P22, a transducing phage discovered more than 50 years ago. Lysogeny with this podovirus results in O1 seroconversion. Many of the phages from the $S$. Typhimurium phage typing scheme were shown to be related to P22 (Schmieger, 1999). The goal of this work was to characterize the SETPs and the relationships between them in some detail with a view to improving our understanding of the basis of phage typing.

\section{METHODS}

Typing phages. The SETPs, numbered SETP1 to SETP16 inclusive, were kindly provided by Linda Ward, LEP, UK. Phages were propagated using the host-overlay method on a $S$. Enteritidis PT1b isolate (PB406), which is susceptible, in varying degrees, to all 16 typing phages.

Plaque morphology. Phage were diluted to achieve discrete plaques and spotted onto a PB406 lawn on 3 separate days. Plaque and halo diameters from five discrete plaques were measured using sliding callipers. The mean and range of sizes were recorded.

Electron microscopy. Phages were sedimented by ultracentrifugation in a Beckman J2-21 ultracentrifuge using a JA-18 fixed angle rotor for $1 \mathrm{~h}$ at $25000 \mathrm{~g}$. This was followed by two washes in $0.1 \mathrm{M}$ neutral ammonium acetate. Particles were deposited on Athene-type copper grids with carbon-coated Formvar films, and stained with $2 \%$ potassium phosphotungstate ( $\mathrm{pH} 7.2$ ) or $2 \%$ uranyl acetate ( $\mathrm{pH} 4.5)$, and examined with a Philips EM electron microscope operated at $60 \mathrm{kV}$. Magnification was monitored with T4 phage tails. Phages were measured on high-magnification micrographs $(\times 297000)$. Twenty intact particles per morphotype were measured. Electron microscopy of the phage samples was performed by Professor Hans Ackermann at the Felix d'Herelle Reference Center for Bacterial Viruses, Universite Laval, Quebec, Canada.

Adsorptions. Phages were incubated for $10 \mathrm{~min}$ with lateexponential-phase cultures of group B $(\mathrm{O} 4,12)$ (S. Typhimurium MS60), D1 (O 9, 12) (S. Enteritidis MS23, S. enterica serovar Panama 683/01), D2 (O 9, 46) (S. enterica serovar Ouakam 494/01, S. enterica serovar Hillingdon 592/01) Salmonella serotypes, Escherichia coli ATCC 25922 and purified LPS from S. Enteritidis $(9,12)$ and S. enterica serovar Minnesota (S. Minnesota) (O21) (Sigma). The approximate number of p.f.u. of each typing phage before and after adsorption was estimated by spotting $7 \mu$ phage suspension onto a PB406 lawn, incubating at $37{ }^{\circ} \mathrm{C}$ overnight and counting the plaques formed.
PFGE of phages. Agarose plugs of intact phages were prepared using the PulseNet PFGE protocol (Ribot et al., 2006). Phage DNA was run without digestion in a $1 \%$ gel in a CHEF Mapper using the autoalgorithm settings of $20-60 \mathrm{~kb}$ for $17 \mathrm{~h}$. Genome size was estimated by comparison with the genome of phage C2 (22 $163 \mathrm{bp})$, P22 (41 $724 \mathrm{bp}$ ) and ES18 (46150 bp) using BioNumerics software.

Extraction of phage DNA, restriction analysis and Southern blotting. DNA was prepared using a modification of the Qiagen lambda midi kit with phenol/chloroform rather than Qiagen columns used to extract the DNA. The DNA was precipitated using 2-propanol followed by two washes with $70 \%$ ethanol, and reconstituted in TrisEDTA buffer.

Phage DNA was digested with the HindIII enzyme. Fragments were separated in a $0.7 \%$ agarose gel for $1.5 \mathrm{~h}$ at $100 \mathrm{~V}$. Digoxigeninlabelled DNA molecular mass marker II (Roche) was used as a size marker. Banding patterns were analysed using BioNumerics software. DNA was transferred from the gel to positively charged nylon membranes (Roche) according to standard protocols (https:// www.roche-applied-science.com/sis/lad/hyb.jsp). The membranes were probed consecutively with digoxigenin-labelled probes to specific genes (Supplementary Table S1 available with the online journal) with stripping of the nylon membrane using dimethylformamide following each individual probe.

PCR amplification and DNA sequencing. Consensus primers were designed to detect genes homologous to those sequences present in either P22 or SETP3 (Supplementary Tables S2 and S3 available with the online journal). Initial primers were based on sequences within the ORF. If products were obtained with internal primers, new primers based on P22 or SETP3 sequences upstream and downstream of the ORF were designed to allow amplification of the entire ORF. Primers were designed using the primer design software packages Primer3 (http://frodo.wi.mit.edu/) (Rozen \& Skaletsky, 2000) and Web Primer (Stanford) (http://seq.yeastgenome.org/cgi-bin/web-primer). The composition of each $50 \mu \mathrm{l}$ PCR was as follows: $35.75 \mu \mathrm{l}$ water for injection (Braun), $5 \mu \mathrm{l} 10 \times \mathrm{Mg}$-free buffer $(500 \mathrm{mM} \mathrm{KCl}, 100 \mathrm{mM}$ Tris-HCl, $1 \%$ Triton X-100) (Promega), $4 \mu \mathrm{l}$ dNTP (200 $\mu \mathrm{M}$ each) (Promega), $3 \mu \mathrm{MgCl}_{2}(1.5 \mathrm{mM})$ (Promega), $1 \mu \mathrm{l}$ primer $(200 \mathrm{nM})$, $0.25 \mu \mathrm{Taq}$ polymerase (1U) (Promega) and $1 \mu$ phage DNA template $\left(0.02 \mu \mathrm{g} \mu \mathrm{l}^{-1}\right)$. PCR amplification was performed in a thermal cycler (I-Cycler; Bio-Rad) with an initial denaturation at $94{ }^{\circ} \mathrm{C}$ for $1 \mathrm{~min}$, followed by 30 cycles of $94{ }^{\circ} \mathrm{C}$ for $45 \mathrm{~s}$, incubation at the annealing temperature for each primer pair for $45 \mathrm{~s}$ and $72{ }^{\circ} \mathrm{C}$ for $1 \mathrm{~min}$, and finishing with a final extension at $72{ }^{\circ} \mathrm{C}$ for $1 \mathrm{~min}$ and storage at $4{ }^{\circ} \mathrm{C}$ thereafter. Amplified products were subjected to electrophoresis on $1 \%$ agarose gels in $0.5 \times$ Tris/borate/EDTA buffer $(45 \mathrm{mM}$ Tris base, $45 \mathrm{mM}$ boric acid, $1 \mathrm{mM}$ EDTA, $\mathrm{pH} 8$ ), stained with ethidium bromide, visualized under UV irradiation and the gels photographed with a Polaroid camera. PCR products were purified using a QIAquick PCR purification kit (Qiagen). Sequencing reactions were performed by Sequiserve.

Isolation of PB406-typing phage lysogens. Dilutions of typing phages were spotted onto a PB406 lawn and incubated overnight. Opaque lysis or regrowth colonies in the phage inoculum were selected with a straight wire, inoculated into double-strength nutrient broth, incubated overnight and subcultured to nutrient agar and XLD (xylose-lysine-desoxycholate) plates. Five colonies from each phage tested were further subcultured onto nutrient agar. Slide agglutination using $\mathrm{O} 1, \mathrm{O} 4$ and $\mathrm{O} 9$ antisera was performed on all potential lysogens. All smooth isolates were inoculated into double-strength nutrient broths and incubated, with shaking at $37{ }^{\circ} \mathrm{C}$ overnight. A $1 \mathrm{ml}$ culture was centrifuged and the supernatant was added to a microfuge tube containing toluene. A $7 \mu \mathrm{l}$ volume was spotted onto a lawn of PB406 and incubated overnight. Plaque formation indicated 
that phage had been induced from the isolate, confirming that it was a lysogen. Phage typing, serotyping with $\mathrm{O} 1$ and PFGE were performed on all confirmed lysogens.

Susceptibility of non-Enteritidis Salmonella to typing phage. Phage typing was performed on a selection of non-Enteritidis isolates from Salmonella group B $(\mathrm{O} 4,12)(n=18), \mathrm{D} 1(\mathrm{O} 9,12)(n=9)$, group $\mathrm{C}(\mathrm{O} 6,7)(n=1), \mathrm{O} 11(n=1)$ and $\mathrm{O} 35(n=1)$ serovars. If an isolate was susceptible to any of the typing phages then phage typing was performed on a further nine isolates of this serovar, if available (Supplementary Table S4 available with the online journal).

Testing of S. Enteritidis isolates for prophage. Slide agglutination with $\mathrm{O} 1$ antiserum (Statens) was performed on $50 \mathrm{~S}$. Enteritidis isolates of a variety of PTs $(n=30)$. The isolates were inoculated into double-strength nutrient broth and incubated with shaking at $37{ }^{\circ} \mathrm{C}$ for 2 days. Four microlitres of mitomycin $\mathrm{C}\left(1 \mathrm{mg} \mathrm{ml}^{-1}\right)$ was added to each broth and the broth was then reincubated for a further $3 \mathrm{~h}$. Cultures were centrifuged with toluene and spotted onto PB406 lawns. Plaque formation indicated the presence of prophage. Adsorption studies using isolates of different serovars were performed on induced prophages as previously described.

Nucleotide sequence accession numbers. Genes assigned accession numbers in the GenBank database were given the following accession numbers: EF151167-EF151189 and EF212157-EF21277 and are listed in Supplementary Table S5 (available with the online journal).

\section{RESULTS}

\section{Electron microscopy}

Phages belonged to three morphotypes and three species only (Figs 1,2 and 3). Phages 2, 4, 6 and 9 were family Myoviridae, species Beccles. Heads measured $60.7 \mathrm{~nm}$ between opposite apices and were icosahedral. Noncontracted tails were $157 \times 17 \mathrm{~nm}$, and had a neck of $7 \times 7 \mathrm{~nm}$ and a sheath of $150 \times 17 \mathrm{~nm}$, with cross striations of about $4 \mathrm{~nm}$ periodicity. There were no collars or baseplates. Contracted tails consisted of a sheath of $57 \times 20 \mathrm{~nm}$ and showed a core of about $155 \times 7 \mathrm{~nm}$.

Phages 3, 5, 7, 11, 12 and 13 were family Siphoviridae, species Jersey. Heads measured $62.5 \mathrm{~nm}$ and were icosahedral. Tails were rigid, non-contractile, measured $120 \times 7 \mathrm{~nm}$, had striations with a periodicity of $4 \mathrm{~nm}$, and carried a $20 \mathrm{~nm}$ wide baseplate with spikes.

Phages 1, 8, 10, 14, 15 and 16 were family Podoviridae, species P22. Heads measured $62.5 \mathrm{~nm}$ and were icosahedral. Tails were $13 \mathrm{~nm}$ long and carried a conspicuous $20 \mathrm{~nm}$ wide base plate with three prongs in profile.

\section{Adsorption to lipopolysaccharide}

All the typing phages, with the exception of Myoviridae SETP2, 4 and 9 were adsorbed by group $\mathrm{B}(\mathrm{O} 4,12)$ and D1 (O 9, 12) Salmonella and not by group D3 (O 9, 46) Salmonella. Similarly all but phage SETP 2, 4 and 9 were adsorbed by lipopolysaccharide purified from $S$. Enteritidis $(\mathrm{O} 9,12)$ but not with lipopolysaccharide purified from $S$. Minnesota (O 21). The results were consistent with adsorption to the $\mathrm{O} 12$ antigen. (a)

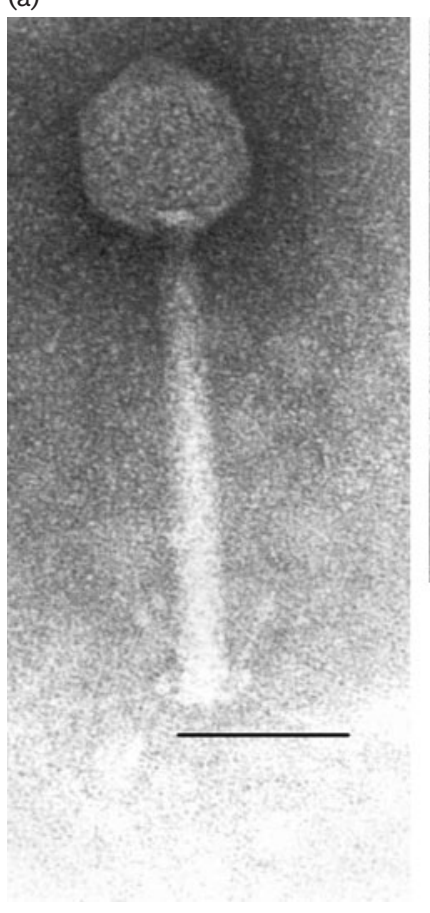

(b)

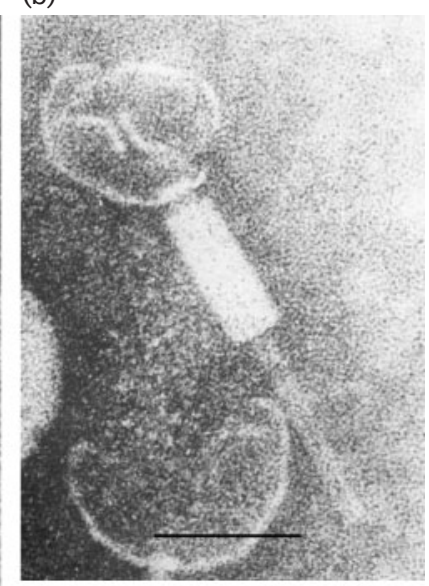

Fig. 1. Morphotype 1. Electron micrographs showing phages stained with $2 \%$ potassium phosphotungstate and photographed at $\times 297000$ magnification. These micrographs show SETP4, from the family Myoviridae, species Beccles, with its tail in the noncontracted (a) and contracted (b) states. Bar, $60 \mathrm{~nm}$.

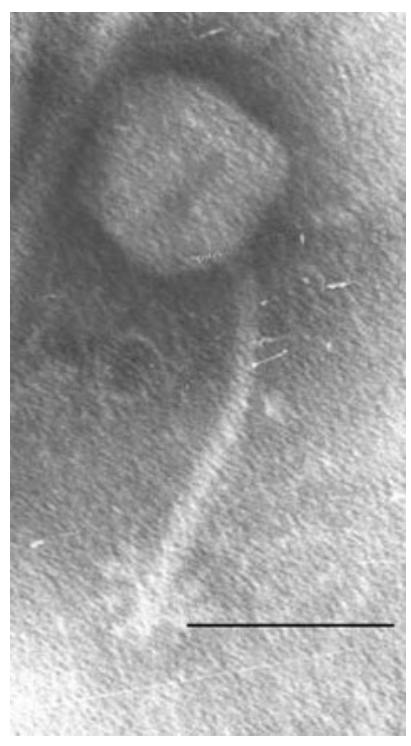

Fig. 2. Morphotype 2. Electron micrograph showing SETP12, from the family Siphoviridae, species Jersey. Bar, $60 \mathrm{~nm}$. 


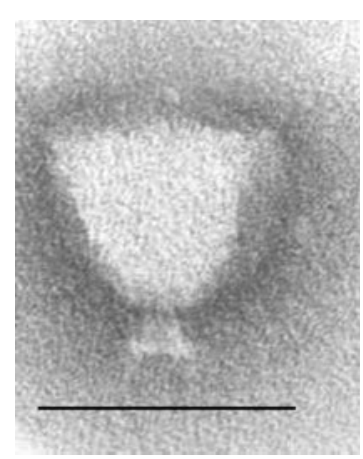

Fig. 3. Morphotype 3. Electron micrograph showing SETP1 from the family Podoviridae, species P22. Bar, $60 \mathrm{~nm}$.

\section{Plaque morphology}

Myoviridae produced uniformly small $(\leqslant 1 \mathrm{~mm})$ plaques without haloes (zone of clearance around each plaque). Podoviridae produced plaques with haloes. Plaques varied from pinpoint $(\mathrm{Pc})$, to $1 \mathrm{~mm}$ diameter $(\mathrm{Pa})$, and to about $3 \mathrm{~mm}$ diameter $(\mathrm{Pb})$. Siphoviridae produced plaques of variable size with or without halo effect.

\section{Genome size and HindIII digestion pattern}

The Podoviridae and Siphoviridae (PFGE-A) were approximately $42 \mathrm{~kb}$ while there was size variation among the Myoviridae. SETP2, 4 and 9 (PFGE-B) were about $36.5 \mathrm{~kb}$ and SETP6 (PFGE-C) was approximately $27 \mathrm{~kb}$. HindIII digestion of phage DNA produced 9 distinct patterns of 8 to 11 bands that showed correlation with morphotype (Fig. 4). The Podoviridae subdivided into three groups, $\mathrm{Pa}$ (SETP1, 8 and 10), Pb (SETP15 and 16) and Pc (SETP14). Likewise the Siphoviridae subdivided into Sa (SETP3, 5 and 12), Sb (SETP7 and 11) and Sc (SETP13), and the Myoviridae also divided into three groups, Ma (SETP4 and 9), Mb (SETP2) and Mc (SETP6).

\section{PCR and sequencing}

Amplification targeting P22 gene homologues produced amplicons only from Podoviridae, and Podoviridae of a given HindIII digestion group yielded a uniform pattern of P22 homologues (Table 1). Oligonucleotide probe hybridization for P22 gene homologues confirmed the results obtained with amplification with one exception. Weak positive reactions were obtained with $\mathrm{Pb}$ phages with $\operatorname{nin} G$ (a)

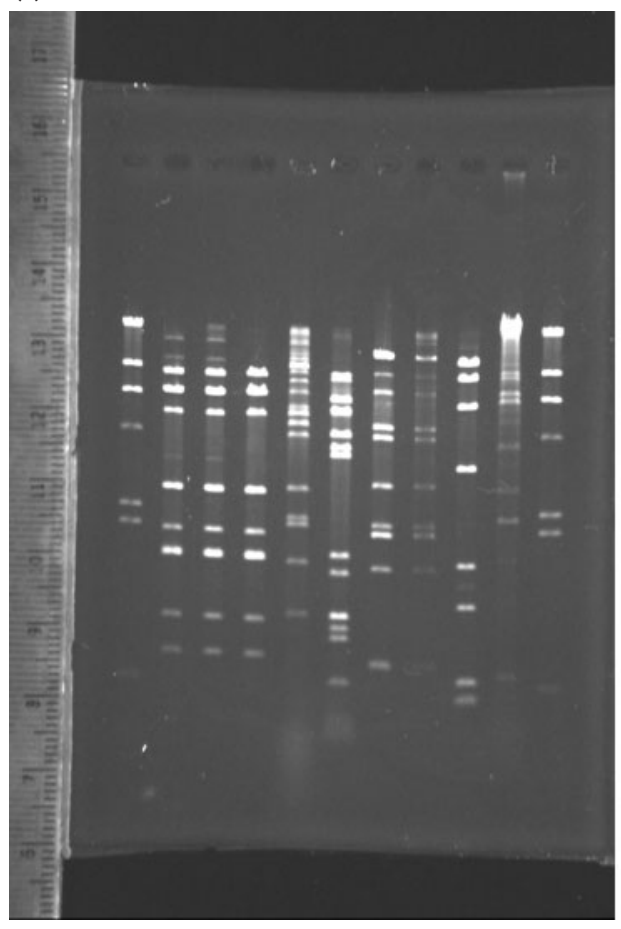

(b)

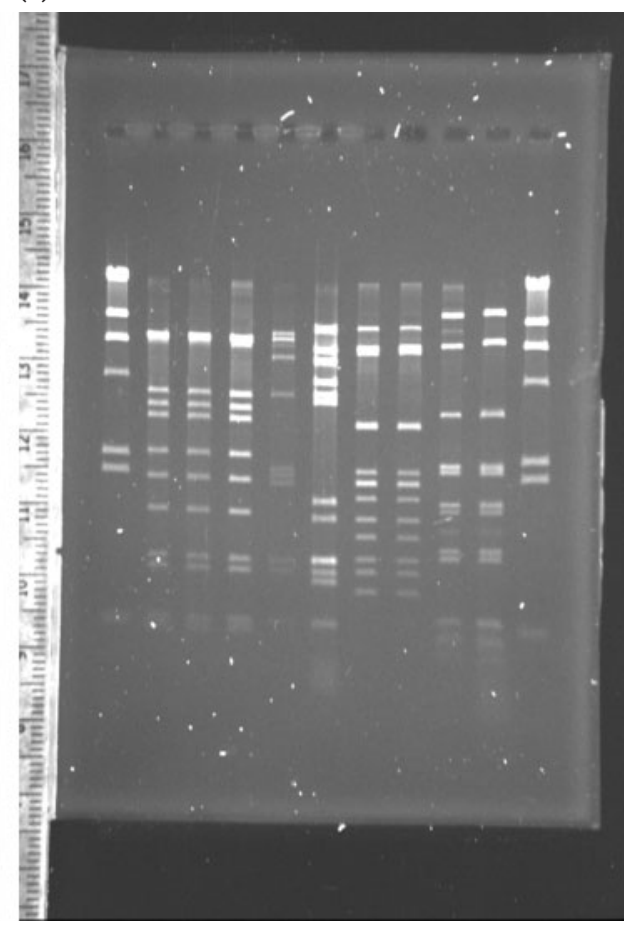

Fig. 4. Hindlll digestion of typing phages. Purified phage DNA was digested with Hindlll and fragments separated by electrophoresis in a $0.7 \%$ agarose gel for $1.5 \mathrm{~h}$ at $100 \mathrm{~V}$. (a) Lane 1, control; lane 2, SETP3; lane 3, SETP5; lane 4, SETP12; lane 5, SETP13; lane 6, P22; lane 7, SETP7; lane 8, SETP11; lane 9, SETP2; lane 10, SETP6; lane 11, control. (b) Lane 1, control; lane 2, SETP1; lane 3, SETP8; lane 4, SETP10; lane 5, SETP14; lane 6, P22; lane 7, SETP15; lane 8, SETP16; lane 9, SETP4; lane 10, SETP9; lane 11, control. 
Table 1. PCR and hybridization results for $P 22$ homologues

The PCR and hybridization results correlated for all phages except for weak positive reactions with $\operatorname{nin} G$ probes. The $\mathrm{Pb}$ group of phages may have a gene similar to $\operatorname{nin} G$ but with insufficient similarity at the nin $G$ primer binding sites to produce amplicons. $\mathrm{w}+$, Weak positive.

\begin{tabular}{|lll|}
\hline Gene & \multicolumn{1}{c|}{ PCR +} & Hybridization + \\
\hline gene9 & $\mathrm{Pa}, \mathrm{Pb}$ and $\mathrm{Pc}$ & $\mathrm{Pa}, \mathrm{Pb}$ and $\mathrm{Pc}$ \\
gene3 & $\mathrm{Pa}, \mathrm{Pb}$ and $\mathrm{Pc}$ & $\mathrm{Pa}, \mathrm{Pb}$ and $\mathrm{Pc}$ \\
gene23 & $\mathrm{Pb}$ & $\mathrm{Pb}$ \\
ninG & $\mathrm{Pa}$ & $\mathrm{Pa}$ and $\mathrm{Pb}(\mathrm{w}+)$ \\
sieB & $\mathrm{Pa}, \mathrm{Pb}$ and $\mathrm{Pc}$ & $\mathrm{Pa}, \mathrm{Pb}$ and $\mathrm{Pc}$ \\
c2 & None & None \\
sieA & None & Not tested \\
ant & None & Not tested \\
gene7 & None & Not tested \\
\hline
\end{tabular}

probes, while amplicons were not produced with ninG primers. PCR for detection of SETP3 gene homologues produced amplicons only with the phages identified as Siphoviridae, and the pattern of SETP3 gene homologues from each phage correlated with the HindIII digestion pattern (Table 2).

For both Podoviridae and Siphoviridae, the sequence of amplified genes was generally uniform for phages within a given HindIII digestion group. The only exception was a single nucleotide at position 5046 in the tailspike-encoding gene of Siphoviridae group Sa, which was different in each of the three members of the Sa group. Sequence variation between homologous genes from distinct HindIII digestion

Table 2. Results of PCR for SETP3 gene homologues

Some of the genes were too large to amplify and sequence in one piece so primers were designed to amplify overlapping regions, e.g. terminase (Term)-, tailspike (Tspk)- and DNA polymerase (DNAP)-encoding genes.

\begin{tabular}{|lccc|}
\hline \multirow{2}{*}{$\begin{array}{l}\text { Protein encoded } \\
\text { by gene }\end{array}$} & \multicolumn{3}{c|}{ HindIII digestion group } \\
\cline { 2 - 4 } & Sa & Sb & Sc \\
\hline HTH & + & - & - \\
Lysozyme & + & - & - \\
Term-1 & + & + & + \\
Term-2 & + & + & $-(+)$ \\
Tspk-1 & + & + & + \\
Tspk-2 & + & + & + \\
Tspk-3 & + & + & + \\
DNAP-1 & + & $-(+)$ & - \\
DNAP-2 & + & - & - \\
DNAP-3 & + & - & - \\
DNAP-4 & + & + & $-(+)$ \\
& + & & \\
\hline
\end{tabular}

+ , PCR positive; - , PCR negative; $-(+)$, PCR negative on first test but positive on second test; $\mathrm{w}+$, weak positive. groups was noted. These sequences were identical or similar to a variety of phage or bacterial gene sequences previously described (Supplementary Table S6 available with the online journal).

\section{Formation of lysogens}

S. Enteritidis PB406-lysogens were formed with the group a (SETP 4 and 9) and c (SETP 6) Myoviridae, and with group a (SETP 1, 8 and 10) and group b (SETP 15 and 16) Podoviridae. Lysogens formed with SETP8 and 10 (group $\mathrm{Pa}$ ) were unstable and could not be successfully propagated. Lysogens were not formed with any of the Siphoviridae. Lysogenization with Podoviridae resulted in antigenic conversion of $S$. Enteritidis PB406 to O1 positive, while no change was detected in the antigenic structure of Myoviridae lysogens. Lysogenization with the typing phages resulted in changes to the isolates phage typing pattern. The lysogen was no longer susceptible to the lysogenic phage, other phages of the same HindIII digestion group and sometimes to other typing phages (results not shown).

PFGE with $X b a$ I showed that all three stable podovirus lysogens had a band at $440 \mathrm{~kb}$. The lysogens formed with the group a Myoviridae (SETP4 and 9) showed an additional band at $31 \mathrm{~kb}$. The banding pattern of the SETP6 lysogen was indistinguishable from the PB406 parent strain (Fig. 5).

\section{Susceptibility of non-Enteritidis Salmonella to typing phages}

All 11 S. enterica serovar Dublin (S. Dublin) isolates (group D1) were susceptible to phage SETP6 and one was

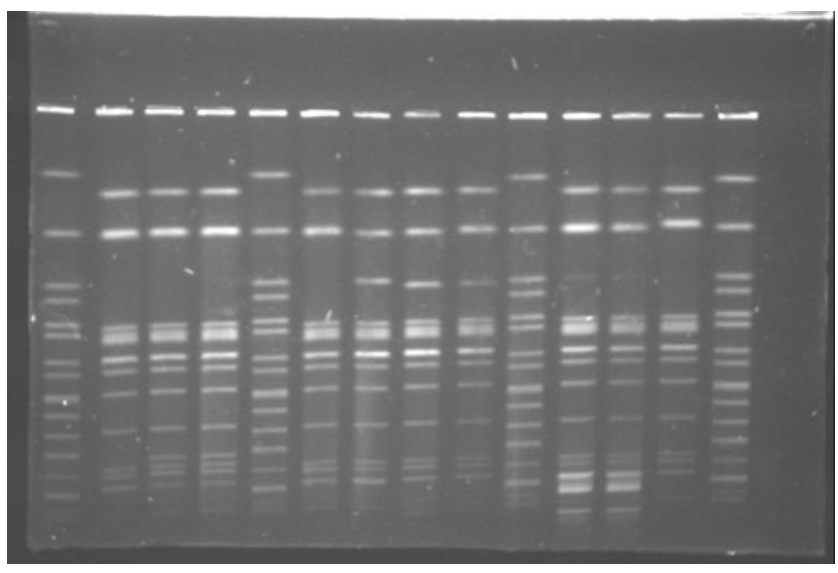

Fig. 5. PFGE of PB406-typing phage lysogens. PFGE of the PB406-lysogens using the PulseNet PFGE protocol with $X$ bal as the restriction enzyme. Lane 1, H9812; lane 2, PB406; lane 3, PB406-SETP4 lys; lane 4, PB406-SETP9 lys; lane 5, H9812; lane 6, PB406; lane 7, PB406-SETP1 lys; lane 8, PB406-SETP15 lys; lane 9, PB406-SETP16 lys; lane 10, H9812; lane 11, PB406SETP8 lys; lane 12, PB406-SETP10 lys; lane 13, PB406-SETP6 lys; lane 14, H9812. 
additionally susceptible to SETP14 (group Pc). Susceptibility of $S$. Dublin to SETP6 is a recognized trait of this serovar (L. Ward, personal communication). Individual members of other serovars reacted with individual phages or with a small group of related phages; however, no consistent patterns other than that of $S$. Dublin were confirmed (Supplementary Table S4 available with the online journal).

\section{Prophage}

A total of 46 out of $50 \mathrm{~S}$. Enteritidis isolates of various PTs had inducible prophages (Supplementary Table S7 available with the online journal). The induced phages generally produced small plaques without haloes and none were successfully propagated on $S$. Enteritidis PB406 using either the host-overlay or broth methods. All the isolates that had inducible phage tested negative with $\mathrm{O} 1$ antiserum and none of the induced prophages adsorbed to group B (4, $12)$, D1 $(9,12)$ or D3 $(9,46)$ Salmonella isolates. Prophage suspensions were ultracentrifuged and PFGE performed on phages from isolates 495/03 ( $S$. Enteritidis PT1), 613/03 ( $S$. Enteritidis PT1), 997/03 (S. Enteritidis PT1), MS22 ( $S$. Enteritidis PT1), 370/03 (S. Enteritidis PT4), PB395 ( $S$. Enteritidis PT21), 664/03 (S. Enteritidis PT29), PB388 ( $S$. Enteritidis PT34), 24/02 (S. Enteritidis PT1) and 405/03 (S. Enteritidis PT1). Size estimation by PFGE showed that the induced prophage genomes were uniform in size at approximately $40 \mathrm{~kb}$.

\section{DISCUSSION}

These data represent the first detailed phenotypic and genotypic characterization of the 16 phages widely used throughout the world for phage typing of $S$. Enteritidis. The collection comprises six Podoviridae, six Siphoviridae and four Myoviridae. All these morphotypes have previously been observed associated with the genus Salmonella (H. Ackermann, personal correspondence). There is a high degree of consistency in the relationships we have defined among the typing phages based on both phenotypic and genotypic properties (Table 3 ).

Digestion with HindIII subdivided each morphotype into three groups, which most likely represent groups of original and adapted phages. Some of the groups correspond with the published phage life history, e.g. SETP5 was adapted from SETP3. In other cases the relationships are less clear. For example SETP6 is a different morphotype than SETP8 and SETP10, although these three phages have been considered as related (Ward et al., 1987). However, phages SETP8 and SETP10 are closely related to SETP1 (Fig. 6).

Of the six newer phages, SETP 11 and 12 represent adaptation of earlier phages (SETP7 and SETP5, respectively), and SETP16 may have been adapted from SETP15. Assuming that the phages with lower numbers in related groups, e.g. SETP 3, 7 and 15, are the original phages, and that the higher numbers represent later adaptation, it is apparent that adaptation has tended to result in broader host range phages. Given the very limited variation observed in the sequence of the gene encoding the tailspike protein across the phages within a specific group, variation in the adsorption properties of the tail spike protein does not seem a likely explanation for broader host range. An alternative explanation for adaptation to a broader host range in many cases may be increased methylation, or acquisition of some other modification. However, incubation of phage DNA with $\mathrm{McrBc}$, an endonuclease that

Table 3. Summary of characteristics of SETPs

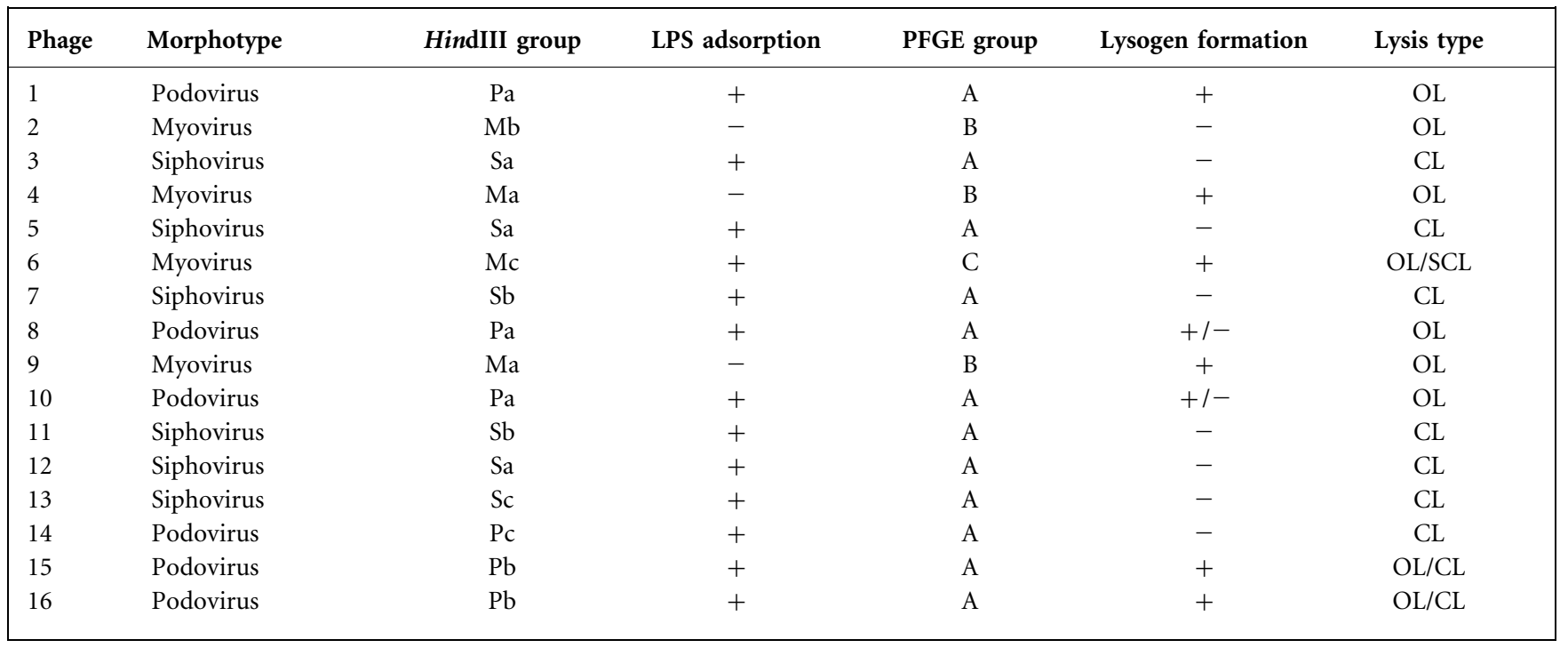

CL, confluent lysis; OL, opaque lysis - indicative of lysogen formation and regrowth; SCL, semiconfluent lysis. 


\begin{tabular}{|c|c|c|c|c|}
\hline Lysogenic & $\Psi 1 *$ & Adapted & $\Psi 8$ & Adapted \\
\hline ? Lysogenic & $\Psi 2$ & & & \\
\hline ? Source* & $\Psi 3$ & Adapted & $\Psi 5$ & \\
\hline Unknown & $\Psi 4$ & Adapted & $\Psi 9$ & \\
\hline Lysogenic & $\Psi 6^{*}$ & & & \\
\hline Sewage & $\Psi 7$ & Adapted & $\Psi 11$ & \\
\hline$?$ & $\Psi 13$ & & & \\
\hline$?$ & $\Psi 14$ & & & \\
\hline ? Lysogenic & $\Psi 15$ & Adapted & $\Psi 16$ & \\
\hline
\end{tabular}

Fig. 6. Proposed history of typing phages. Asterisks indicate where this work differs from the published phage history. The history of the initial 10 typing phages has been published elsewhere. However, this work suggests some different relationships between the phage. SETP1 was indistinguishable from SETP8 and SETP10 suggesting that these phages were most likely adapted from SETP1 and not SETP6. SETP3 is a virulent phage and is therefore unlikely to have been propagated from a lysogenic strain. Some of the more recent phages are indistinguishable from phages in the initial set of 10 and have most likely been adapted from them, e.g. SETP11 from SETP7, and SETP12 from SETP5.

specifically digests $\mathrm{CpG}$ methylated DNA, failed to demonstrate methylation of any of the 16 phage genomes (results not shown). However, as we did not have access to the specific phage propagating strains used at LEP for this study the phages were propagated on S. Enteritidis PB406. Therefore, it is possible that the propagated phages did not have the epigenetic characteristics of the usual typing phages supplied by LEP.

The known plating properties of the typing phages correlate with the morphotypes determined in this study in that all of the typing phages that were characterized by clear lysis patterns were Siphoviridae (which do not form lysogens) while opaque lysis was associated with those phages identified as Podoviridae and Myoviridae.

Previously, differences in phage receptors have been suggested as being a major factor in explaining differences in susceptibility to the SETPs (Guard-Petter, 1999). However, we have demonstrated that all Podoviridae and Siphoviridae adsorb in the presence of the O12 lipopolysaccharide antigen; therefore, variation in adsorption does not appear to be a major factor in the difference of susceptibility of isolates of $S$. Enteritidis to these phages. Loss of lipopolysaccharide is significant in relation to the reported emergence of $\mathrm{PT} 7$, a rough strain of $S$. Enteritidis, from a number of different PTs. Our data indicate that the phages to which PT7 isolates remain susceptible are the Myoviridae SETP2, 4 and 9, which do not adsorb to lipopolysaccharide (presumably they have an alternative receptor), and the unique siphovirus SETP13. SETP13 does adsorb to lipopolysaccharide but may also be able to use an alternative receptor.

This study raises interesting questions regarding the relationship between $S$. Enteritidis and Podoviridae in nature. S. Enteritidis is listed as $\mathrm{O} 1$ positive in the Kaufmann-White scheme and the O1 antigen is known to be encoded on P22-like O1 converting phages. However, no P22-like phages have been detected in the sequenced $S$. Enteritidis genomes (http://www.salmonella.org/genomics/ sen.dbs and http://www.sanger.ac.uk/Projects/Salmonella/ SEN_genepred.embl). None of the $50 \mathrm{~S}$. Enteritidis isolates of various PTs tested in this study were O1 positive. Although some of the six typing Podoviridae readily produced lysogens with PB406 some of these were highly unstable even in laboratory conditions. Furthermore, the characteristics of those prophage that were induced $(46 / 50)$ from $S$. Enteritidis contrasted with the characteristics of the Podoviridae of the typing scheme. The latter adsorb to lipopolysaccharide and produce large plaques with haloes, while the former do not adsorb to lipopolysaccharide from Salmonella and produce pinpoint plaques on PB406. Furthermore, the PFGE patterns associated with the podovirus lysogens generated in this study do not appear in our database of PFGE patterns for wild isolates of $S$. Enteritidis, nor are we aware of reports of this PFGE pattern from elsewhere. In general PFGE patterns of $S$. Enteritidis are much more homogeneous than the patterns observed with $S$. Typhimurium and a number of other serotypes. We speculate that stable podovirus lysogens may form much less readily with $S$. Enteritidis than with $S$. Typhimurium. This contrasts with $S$. Typhimurium where the $\mathrm{O} 1$ antigen is commonly present and several P22-like O1-converting prophages have been identified. This could have significant implications for understanding genetic exchange in $S$. enterica and may be related to the limited diversity observed in PFGE patterns of $S$. Enteritidis.

The Myoviridae in the phage typing scheme were more similar to the prophages detected in $S$. Enteritidis isolates in that they produced small plaques without haloes and did not adsorb to lipopolysaccharide antigens. Myoviridae sequences have been detected in S. Enteritidis, e.g. Fels-2 (Porwollik et al., 2005).

Sequencing results emphasize the mosaicism of the phage genomes. HindIII group Pa (SETP1, 8 and 10 genomes) had a gene9 (tailspike-encoding) sequence identical to that of ST64T, while their sieB sequence was very similar to that of a prophage in S. enterica serovar Paratyphi A (525/ 527 bp identical). Comparison of sequence results with BLASTX, along with phenotypic data, allows prediction of the life cycle of certain of the phages. Both the Podoviridae (gene3) and Siphoviridae (term) had terminase-encoding 
genes that are suggestive of a headful mechanism of packaging DNA. All six Siphoviridae in the typing set had evidence of possessing a DNA polymerase, a characteristic of virulent phages.

Understanding phage-host interactions is important in understanding microbial evolution, and may have more immediate application in developing a molecular equivalent of phage tying, phage therapy or phage-based decontamination of food. This study defining the main characteristics of this widely used group of phages provides a basis for more extensive investigation of the basis of phage-host interactions. The study also suggests a reorganization of the template for application of phages to bacterial lawns in phage typing, as the application of related phages on adjacent spots may facilitate more intuitive reading of patterns of phage reaction. A better understanding of the relationships between the typing phages may permit simplification of the phage typing scheme by elimination of some closely related phages from the scheme.

\section{ACKNOWLEDGEMENTS}

We thank Linda Ward, LEP, Health Protection Agency, UK, for supplying the typing phages for this project. We thank Hans Ackerman, University Laval, Quebec, Canada, for the electron micrographs of the phages. We thank Gayle Philip, National University of Ireland, Maynooth, Ireland, for help with annotation of SETP3.

\section{REFERENCES}

Baggesen, D. L., Wegener, H. C. \& Madsen, M. (1997). Correlation of conversion of Salmonella enterica serovar Enteritidis phage type 1, 4, or 6 to phage type 7 with loss of lipopolysaccharide. J Clin Microbiol 35, 330-333.

Fortier, L. C., Bouchard, J. D. \& Moineau, S. (2005). Expression and site-directed mutagenesis of the lactococcal abortive phage infection protein AbiK. J Bacteriol 187, 3721-3730.

Frost, J. A., Ward, L. R. \& Rowe, B. (1989). Acquisition of a drug resistance plasmid converts Salmonella enteritidis phage type 4 to phage type 24. Epidemiol Infect 103, 243-248.

Guard-Petter, J. (1999). Phage type and other outer-membrane characteristics of Salmonella enterica serovar Enteritidis associated with virulence. In Salmonella Enterica Serovar Enteritidis in Humans and Animals, vol. 1, pp. 221-232. Ames, IA: Iowa State University Press.

Mmolawa, P. T., Schmieger, H., Tucker, C. P. \& Heuzenroeder, M. W. (2003). Genomic structure of the Salmonella enterica serovar Typhimurium DT 64 bacteriophage ST64T: evidence for modular genetic architecture. J Bacteriol 185, 3473-3475.

Porwollik, S., Santiviago, C. A., Cheng, P., Florea, L. \& McClelland, M. (2005). Differences in gene content between Salmonella enterica serovar Enteritidis isolates and comparison to closely related serovars Gallinarum and Dublin. J Bacteriol 187, 6545-6555.

Raes, M., Ward, L. R., Maas, H. M. E., Van Leeuwen, W. J. \& Henken, A. M. (2000). Test Results of Salmonella Sero- and Phage Typing by the National Reference Laboratories and the EnterNet Laboratories in the Member States of the European Union. Collaborative Study IV on Seroand Phage Typing. Rijksinstituut voor Volksgezondheid en Milieu report 284500 013. http://rivm.openrepository.com/rivm/bitstream/ 10029/9440/1/284500013.pdf (http://hdl.handle.net/10029/9440)

Rankin, S. \& Platt, D. J. (1995). Phage conversion in Salmonella enterica serotype Enteritidis: implications for epidemiology. Epidemiol Infect 114, 227-236.

Ribot, E. M., Fair, M. A., Gautom, R., Cameron, D. N., Hunter, S. B., Swaminathan, B. \& Barrett, T. J. (2006). Standardization of pulsedfield gel electrophoresis protocols for the subtyping of Escherichia coli O157:H7, Salmonella, and Shigella for PulseNet. Foodborne Pathog Dis 3, 59-67.

Ridley, A. M., Punia, P., Ward, L. R., Rowe, B. \& Threlfall, E. J. (1996). Plasmid characterization and pulsed-field electrophoretic analysis demonstrate that ampicillin-resistant strains of Salmonella Enteritidis phage type 6a are derived from Salm. Enteritidis phage type 4. J Appl Bacteriol 81, 613-618.

Rozen, S. \& Skaletsky, H. J. (2000). Primer3 on the WWW for general users and for biologist programmers. In Bioinformatics Methods and Protocols: Methods in Molecular Biology, pp. 365-386. Edited by S. Krawetz \& S. Misener. Totowa, NJ: Humana Press.

Schmieger, H. (1999). Molecular survey of the Salmonella phage typing system of Anderson. J Bacteriol 181, 1630-1635.

Tanaka, K., Nishimori, K., Makino, S., Nishimori, T., Kanno, T., Ishihara, R., Sameshima, T., Akiba, M., Nakazawa, M. \& other authors (2004). Molecular characterization of a prophage of Salmonella enterica serotype Typhimurium DT104. J Clin Microbiol 42, 1807-1812.

Vander Byl, C. \& Kropinski, A. M. (2000). Sequence of the genome of Salmonella bacteriophage P22. J Bacteriol 182, 6472-6481.

Ward, L. R. (2006). Salmonella - the last fifty years. In Proceedings of the International Symposium on Salmonella and Salmonellosis, St Malo.

Ward, L. R., de Sa, J. D. \& Rowe, B. (1987). A phage-typing scheme for Salmonella Enteritidis. Epidemiol Infect 99, 291-294. 